\section{A CARDIOSPASM DILATOR}

\section{J. W. LARIMORE, M.D., St. Louis}

The cardiospasm dilator described here was developed to meet certain exigencies in the treatment of cardiospasm cases. It was made by using an ordinary stomach tube of small diameter and attaching to its end the tapering tip of a small bougie. A rubber balloon was then fastened about the tube and bougie end, as shown. About this was placed a bag made of very light weight silk, and so cut that when the first or inner balloon was inflated, there would appear a constriction about the middle. A second balloon was fastened over this. Air-tight fastening of the ends of the balloons was made with rubber cement and winding with small silk thread. This constitutes the dilator proper. Another small balloon may be added inside the first large balloon at its proximal end (Figs. 1 and $2 B$ ). This balloon is also covered by a silk bag which limits its degree of inflation. Its tube for inflation may extend, as is shown, through the lumen of the stomach tube. By a three- dilator. way stopcock on the proxi-

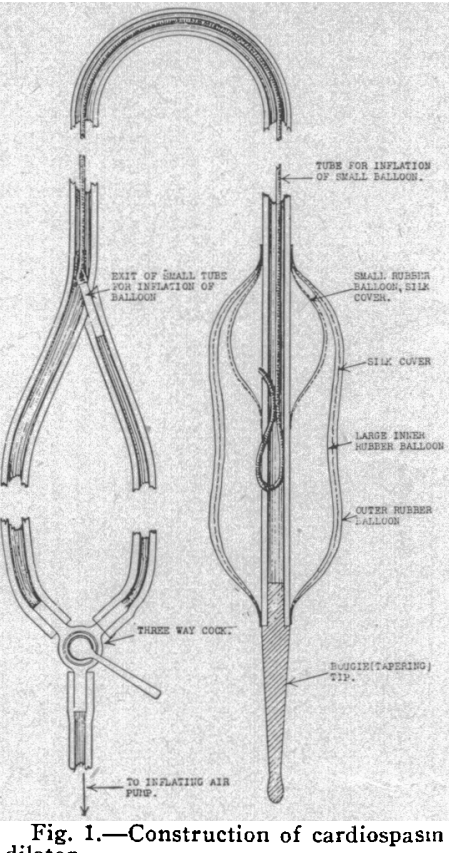
mal end of the apparatus, either the small balloon or the large outer balloons may be inflated. The small balloon is used by inflating it primarily for locating the instrument definitely within the constriction. It may or may not be used, as circumstances demand.

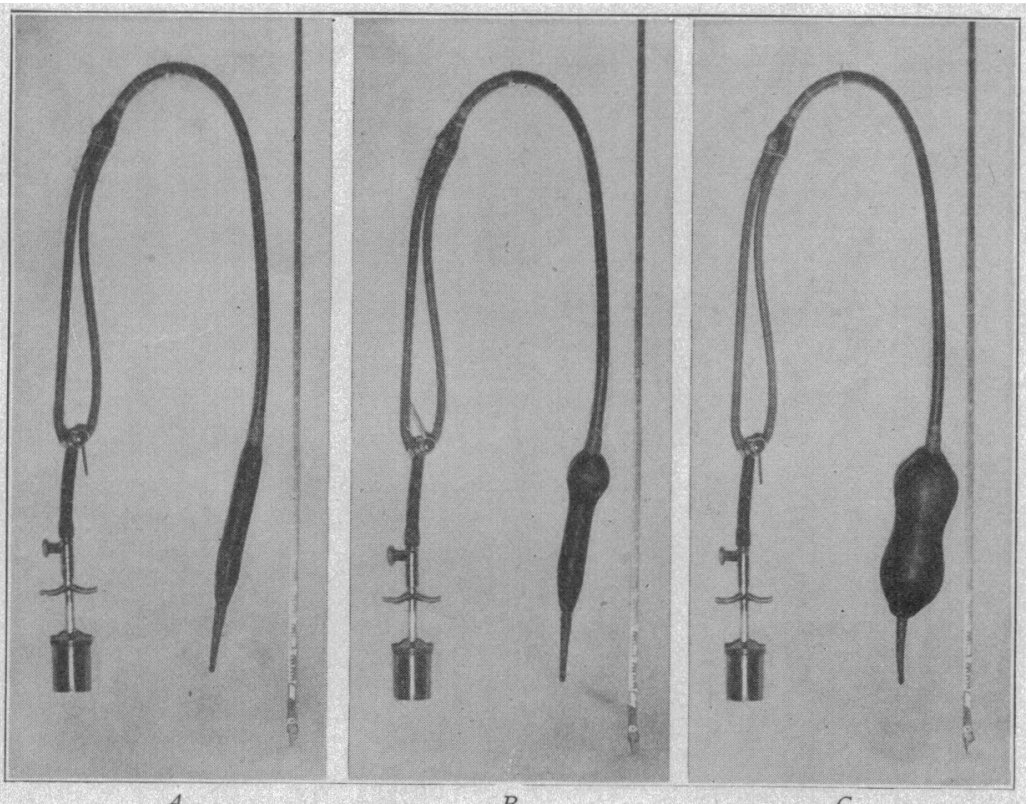

Fig. 2.-Cardiospasm dilator: $A$, uninflated; $B$, with the small inner balloon inflated; when this is introduced to the constriction, the large balloon is properly placed within the spasm; $C$, with the three walled dilating balloon fully inflated. and sure guide. The number of strokes of the pump necessary to secure inflation to a moderate or high pressure may be previously determined, and with this knowledge the extent of dilatation during treatment may be readily estimated by counting the strokes which are sufficient to produce pain reaction.

A mandarin is unnecessary for the introduction of the instrument. The tapered bougie tip makes unnecessary preliminary dilatation with bougies. The construction of the inflation balloons allows the instrument to establish itself at the constriction level without slipping above into the sac or below into the stomach.

\section{PELLAGRA IN CHILE}

Howard K. Tuttle, M.D., Coquimbo, Chile, South America

I believe that this case of pellagra is the first to appear in Chile.

History.-A white girl, aged 11 years, was brought to our company dispensary for treatment. The other members of the family, including three sisters and two brothers, were in good health. The patient first noticed that the skin on the backs of the hands was becoming red, and that the redness was gradually extending up the forearm, being accompanied by considerable burning and itching. Later the disease appeared on the face, forehead and sides of the neck; the tongue was swollen and sore, and the mouth hot and dry. There were pain in the epigastrium, intense thirst and severe attacks of vomiting. Diarrhea accompanied by cramplike pains in the lower abdomen had been present for some time. Nervous symptoms, such as headache, dizziness, choking sensations, tenderness over the spine, or mild convulsions, also disturbed the patient.

Physical Examination.-The skin over the backs of the hands, forearms, face, forehead and sides of the neck was covered with a reddish-brown, scaling, thickened epithelium. On the hands and arms the scaling was very marked, and extended from the base of the nails to within about 3 inches of the elbow; ending in a distinct outline at the dress sleeve. The mucous membrane of the tongue was swollen, reddened and thickened, but smooth, except for a few deep fissures. The patient had a sad, forlorn facial expression. There was some tremor, and a slow, unsteady gait, showing marked weakness. Palpation revealed severe pain over the epigastrium and dorsal spine.

Treatment and Course.-All corn, cornmeal, rice and dried vegetables were prohibited, and meats, fish, eggs, fruits and milk substituted. Arsenic and dilute hydrochloric acid in proper doses were given internally, and an ointment composed of oil of cade, zinc oxid and petrolatum was applied. Under this treatment the dermatitis showed considerable improvement, especially on the face. This treatment had been instituted during the winter months, but when spring returned, the child was again living in the careless condition of poverty and indifference. The disease began to make rapid progress, resulting in extreme emaciation, anemia, mild dementia and death.

\section{COM MENT}

This case presented symptoms so distinct that they could hardly have been mistaken. It is of particular interest, since it serves to illustrate the wide distribution of pellagra, and it is probably the first case reported from Chile.
Air inflation has been found most satisfactory, and is accomplished by the pump from a blood-pressure apparatus. The instrument is shown in the illustrations, and its dimensions may be determined from the accompanying measure.

It has been found that a manometer need not be used, and that the expression of pain by the patient is an adequate
Disease Defined.-Disease is that condition of an individual in which there is, apart from the normal changes due to age, an alteration of the structures or functions or sensations usually found in the average man of any particular race.Prof. Ernest S. Reynolds, Bradshaw Lecture. 